This item was submitted to Loughborough's Research Repository by the author.

Items in Figshare are protected by copyright, with all rights reserved, unless otherwise indicated.

\title{
Variable step radial ordering in carbon onions
}

PLEASE CITE THE PUBLISHED VERSION

http://dx.doi.org/10.1016/j.diamond.2012.11.012

\section{PUBLISHER}

(C) Elsevier B.V.

\section{VERSION}

AM (Accepted Manuscript)

\section{PUBLISHER STATEMENT}

This work is made available according to the conditions of the Creative Commons Attribution-NonCommercialNoDerivatives 4.0 International (CC BY-NC-ND 4.0) licence. Full details of this licence are available at: https://creativecommons.org/licenses/by-nc-nd/4.0/

\section{LICENCE}

CC BY-NC-ND 4.0

\section{REPOSITORY RECORD}

Siklitskaya, Alexandra, Sergey Yastrebov, and Roger Smith. 2019. "Variable Step Radial Ordering in Carbon Onions". figshare. https://hdl.handle.net/2134/16931. 


\title{
Variable step radial ordering in carbon onions
}

\author{
Alexandra Siklitskaya, Sergey Yastrebov *, Roger Smith ${ }^{1}$ \\ A.F.Ioffe Physical-Technical Institute, Department of Solid State Physics, St.Petersburg, \\ 194021, Russia \\ ${ }^{1}$ Loughborough University, Department of Mathematical Sciences, Loughborough, LE11 \\ 3TU, $U K$
}

\begin{abstract}
We analyse the radial distribution of atoms in carbon onions optimised using a combination of classical molecular dynamics and density functional theory. X-ray diffractograms of thermally annealed nanodiamonds are also exploited together with high-resolution transmission electron microscopy data published elsewhere. The internal radial ordering of atoms inside the onions was determined showing a gradual change of intershell separation as a function of radius. This change may result in a twisted internal structure of the onions. The influence of atomic defects appearing in the shells altering their curvature on the formation of twisted onions is discussed.
\end{abstract}

Keywords:

Nanodiamonds, carbon onions, density functional theory, molecular dynamics, X-ray diffraction, high resolution transmission electron microscopy

\footnotetext{
*Corresponding author. Tel/Fax: +7(812)2971017/+7(812)2928983. Email address: yastrebov@mail.ioffe.ru (Sergey Yastrebov)
} 


\section{Introduction}

Open shell Carbon Onions (COs) may be useful structures with many applications such as hydrogen storage, new absorbers and targeted drug delivery vehicles [1] through the encapsulation of other molecules. They might also be precursors that occur in the nucleation of diamond [2]. Modelling the structural properties of $\mathrm{COs}$ is an alternative to expensive laboratory experiments which can throw new light on their geometry and physical and chemical properties. Recently, classical molecular dynamics (MD) simulations were performed by our group to investigate the heating of vacuumsuspended diamond nanocrystals of extremely small size [3] $(1.3-1.4 \mathrm{~nm})$. The spike heating was assumed to occur through the stochastic absorption of ultraviolet photons by the diamond nanoclusters in the interstellar medium [3]. It was found, depending on the heating temperature, that the nanodiamonds transform into nanoclusters with a diversity of shapes not previously mentioned in the literature. Namely, some of them were spirally twisted onions, resembling the structure of the marine mollusk Rapana venosa [3]. These obtained clusters occur in a chiral [3] form.

In this paper the coordinates of carbon atoms obtained by classical MD [3] for a spiral onion are relaxed using Density Functional Theory (DFT), with the code PLATO [4] to test stability. A further aim is the determination of the internal distribution function of atoms inside the cluster, as a function of the radial distance from the centre and to compare the results with experiments. To this end we analyse the most intense X-Ray Diffraction (XRD) peak measured for COs in paper [5] and other results for the variation of the intershell distances measured directly from high resolution 
electron microscopy (HRTEM) images [2].

For the purpose of investigation of the XRD diffraction pattern, we elaborate on a model of broadening of the most intense XRD peak of COs, concentrating on the contribution of the variation of Bragg's angle to the resulting pattern. This broadening may occur due to the atomic ordering within the shells which have a specific distribution of intershell spacing.

\section{Results and discussion}

\subsection{X-ray Diffraction Laboratory Experiment}

The Scherrer equation for the XRD profile, would interpret the smaller broadened peak in Fig. 1 as corresponding to a crystal of bigger size. As is seen from equation 1 , the mean radius of coherent X-ray scattering associated with crystallite size is inversely proportional to the width of the XRD band measured at half its height. However from a comparison of the diffraction profiles of nanodiamonds and onions depicted in Fig.1, this is not the case here. So the broadening mechanism associated with COs cannot be interpreted as due to the size of the onions. Therefore, it seems quite natural to assign the peak broadening to a variation in the "lattice spacings", i.e. the distances between shells of onions. For a more rigorous analysis we shall present a formalism here.

This process of transformation of nanodiamonds to onions occurs with a decrease in the mass density of diamond to the value approximately equal to that of graphite. The transformation results in a corresponding increase in size of the onion child compared to the size of a parent diamond nanocluster.

For example, taking the density of graphite as $\sim 2.2 \mathrm{~g} / \mathrm{cm}^{3}$ and $3.515 \mathrm{~g} / \mathrm{cm}^{3}$ 
for diamond (see, e.g. [6]) we have for a parent nanodiamond with mean diameter $5 \mathrm{~nm}$, a diameter of $\sim 6 \mathrm{~nm}$ for a median child onion.

If there was a strong correspondence between the atomic spacing in the COs and the nanodiamonds, the XRD pattern formed due to the size distribution of the onions only would be similar to that for nanodiamonds (Fig.1) and the shape of the peak of the diffraction pattern would be a rather sharp.

However, experiments show (see, e.g. [7], [8]) that this transformation is accompanied with a significant broadening of the most intense XRD peak after annealing, compared to the peak for pristine nanodiamond (Fig.1).

Excepting the contribution due to internal strain, the broadening of the diffraction pattern can occur through two basic mechanisms, namely broadening induced by the finite size of the nanoclusters and broadening induced by the variation in the lattice (intershell) spacings inside the clusters. From the well-known Scherrer formula the mean crystallite size, $\hat{D}$, is:

$$
\hat{D}=\frac{K \lambda}{w_{L} \cos \left(\psi_{B}\right)} \approx \frac{\lambda}{w_{L}}
$$

here $\lambda$ is wavelength of the $\mathrm{X}$-ray radiation, $w_{L}$ is the full width of the contour measured at half height, $K \approx 1$ for spherical particles, $\cos \left(\psi_{B}\right) \approx 1$ because of the smallness of the Bragg angle $\psi_{B}$ taken for $n=1$ from Bragg's law:

$$
2 \delta r \sin \psi_{B}=n \lambda
$$

where $\delta r$ is lattice spacing.

For investigation of the line shape of the most intense diffraction peak of COs we have used two models. The first is based on an assumption that a set of uniform spheres with a certain size -distribution function may contribute to the diffraction pattern. 
The second one assumes a stochastic contribution of intershell distances to the pattern.

The principles of the first model are presented in paper [9] where direct calculation of the diffraction intensity profile by an ensemble of spherical particles with log-normal distributed sizes was performed and it was shown that for the case of a rather narrow distribution function of cluster sizes, the XRD profile associated with this distribution follows the Lorentzian (Cauchy) function [9]:

$$
L(k)=\frac{2 \hat{A}_{L}}{\pi} \frac{\hat{w}_{L}}{4 k^{2}+\hat{w}_{L}^{2}}
$$

Here $k$ is the wavenumber associated with the diffraction vector, $k=\frac{2\left(\sin \psi-\sin \psi_{B}\right)}{\lambda}$, $\hat{w}_{L}$ is the FWHM. Assuming a small angle of diffraction, one may write $k \approx k^{\prime}=\frac{\left(2 \psi-2 \psi_{B}\right)}{\lambda}$ and rewrite equation (3) as follows:

$$
L\left(\psi, \psi_{B}, w_{L}, A_{L}\right) \approx \frac{2 \hat{A}_{L}}{\pi} \frac{\frac{w_{L}}{\lambda}}{k^{\prime 2}+\frac{w_{L}^{2}}{\lambda^{2}}} \approx \frac{2 A_{L}}{\pi} \frac{w_{L}}{4\left(2 \psi-2 \psi_{B}\right)^{2}+w_{L}^{2}}
$$

here $\quad \hat{w}_{L}=\frac{w_{L}}{\lambda} ; \hat{A_{L}}$ and $A_{L}$ are constants.

The results of fitting the XRD profile for nanodiamonds using equation (3) is shown in Fig.2 by the full line with the fitting parameters in the figure caption. Because the Bragg angles may fluctuate in the COs, it is natural to perform a decomposition of the most intense diffraction peak using the equation:

$$
P(\psi) \approx \sum_{j=1}^{M} L\left(\psi, \psi_{B j}, w_{L j}, A_{L j}\right)
$$

Because there should be a strong correlation between the diamond and onion distributions, this correlation justifies the choice of Lorentzians in equation 
(5) keeping the same parameter $w_{L j}$ but correcting it taking into consideration a slight inflation of size of the onions as a result of annealing. I.e., because this parameter defines the median diameter of the nanocrystallite, we slightly reduced its value for onions, taking into consideration the reduction of density during the diamond-onion transformation similar to that for diamond-graphite transformation. This decreases the number of fitting parameters in equation 5 . We found that fitting with an odd number of Lorentzians (we tried seven and eleven Lorentzians) results in decomposition with positive amplitudes. The upper number of Lorentzians was limited by a reasonable error value generated in the course of application of the leastsquares method.

The results of fitting together with values of the fitting parameters are presented in the Figure 2 captions for seven Lorentzians involved in the fit $(M=7$ in the equation (5)). The fit with $M=11$ is not presented for eye convenience to avoid overload with the contour lines. It is possible to demonstrate that the trend in the order and intensity of the maxima of the Lorentzians does not depend on the number of Lorentzians involved in the decomposition.

We also used a stochastic model for analysis of the contour profile. Only two Gaussians were considered in the decomposition of the experimental data, as follows:

$$
P(\psi)=\sum_{i=1}^{2} \frac{A_{i}}{w_{i} \sqrt{\frac{\pi}{2}}} e^{-2 \frac{\left(\psi-\psi_{o i}\right)^{2}}{w_{i}^{2}}}
$$

$\psi_{o i}$ is the most probable diffraction angle; $w_{i}$ equals 2 times the standard deviation of the Gaussian distribution $\left(2 \sigma_{i}\right)$ or approxi- 
mately 0.849 the width of the peak with number $i$ measured at half height. The result of fitting of equation (6) to the experimental data in the actual range of the diffraction angles is presented in Fig. 2 by dotted and dash-dotted curves with the best fit parameters given in the Fig.2 captions. It is seen from Fig.2 that the model fits the experimental data reasonably well. Note that the resulting profile demonstrates a lack of symmetry.

After analysis of HRTEM images of COs, some authors concluded that the intershell spacings for the onions lie within the range $3.5-3.8 \AA$ [4]. This estimation is in a good agreement with the position and width of the Gaussian marked by dotted-dashed line in Fig.2.

The X-ray diffraction profiles given in Fig.2 exhibit a lack of symmetry. Therefore application of the standard procedure for fitting the diffraction peak with a Voigt function is inappropriate since the Voigt profile is symmetric. Thus, standard procedures of analysis of the internal strain cannot be applied for the case of CO. Moreover, for the $\mathrm{CO}$, the other X-ray diffraction peaks are weak in comparison. This poor resolution of the line shape for the rest of the bands complicates the analysis. Therefore, one might fail with application of standard methods allowing separation of the size and strain parts in the diffraction pattern broadening. The asymmetry of the diffraction peak which gives a pronounced increase in the diffraction intensity, towards the smaller angular region (Fig.1, Fig.2), may result from a spiral structure of COs forming the diffraction pattern. 


\subsection{Numerical Experiment}

It is also possible to perform a similar comparison with data obtained from numerical computation.

Previous work using classical MD [3] had determined that carbon spiral COs could be formed in the course of thermal annealing of nanodiamonds. Here we relaxed some of the resulting structures using DFT but we found that the optimisation by DFT does not introduce significant changes to the cluster topology.

In this paper we thoroughly calculated the variation of the interlayer distance in the numerically DFT- optimised CO structures as a function of the radial distance from the centre of the onion, for the direct comparison with the available experiment. For this purpose we exploited the equatorial cross sections of the $\mathrm{CO}$ obtained by DFT. Typical images of a twisted $\mathrm{CO}$ and its equatorial cross section (Fig.3a) are presented in Fig.3 and Fig.4, correspondingly. We measured the intershell distance as a function of the radius, varying the equatorial angle. The origin of the coordinate system was selected at the centre of the cross-section. Fig. 4 shows the spiral, which was drawn for convenience as an artist impression, to fit the internal distribution of atoms inside the CO. It is seen in the picture that the spiral circles a compact, mainly $s p^{3}$-bonded core in the $\mathrm{CO}$ centre. For calculation of the dependence of the intershell distance on the radius, a line was drawn connecting the $\mathrm{CO}$ centre of the cross section and points on the adjacent spiral turns, for a fixed equatorial angle. Afterwards, the equatorial angle was 
changed and the calculation was repeated for the new set of crossing points. The scatter of points depicted in Fig.4 about the spiral introduces error bars in Fig.5 where the results are presented by the grey circles, where the symbol $R$ denotes the normalised $\mathrm{CO}$ radius calculated using the equation:

$$
R=\frac{r-r_{\text {core }}}{r_{\max }}
$$

here $\mathbf{r}$ is radial distance inside the $\mathrm{CO}\left(r_{\max } \geq r \geq r_{\text {core }}\right), r_{\text {core }}$ is radius of the core and $r_{\max }$ equals to the external onion radius with subtraction of the core radius for convenience of comparison with the laboratory experiment data.

Fig. 5 shows the full dependence of $R$ as a function of the intershell spacing where the lines connecting the data are given for eye guidance only. It is seen from Fig.5, that the dependence attains saturation at $\delta r \sim 3.3 \AA$. The uncertainty in the positions of the atoms is marked by bars. The origin of the bars arises from a statistical averaging of intershell distances measured for different equatorial cross sections. The origin of the uncertainty may be associated with presence of internal strain in the $\mathrm{CO}$, resulting in stochastic fluctuation of atoms about their optimal positions. The origin of the strain results from distorted bonds, their torsions, and fluctuations of valence angles appearing within the shells of the CO. These defects may cause a symmetry break in the trigonal environment of carbon atoms belonging to the strained CO's shell. As a result, carbon atoms may move from their optimal positions in an 'ideal' CO shell. The data presented in Fig.5 may be used for 
estimation of the strain-induced radial displacement of atoms to the X-ray diffraction peaks broadening. It is seen from the figure that the value of the error associated with irregularities in the position of atoms scattered about the ideal spiral trajectory does not exceed $5 \%$ of the absolute value of the intershell spacing. Thus, one may expect, the contribution of the displacement should not exceed $5 \%$ of the mean value of the corresponding Bragg angles (see equation (2)).

\subsection{Comparison between the Numerical and Laboratory Experiments}

\subsubsection{TEM data}

Comparing X-ray diffraction and high resolution HRTEM enables direct observation of the intershell distances in the CO. So, in addition a comparison of the results of COs subjected to simultaneous annealing and electron irradiation observed in HRTEM is also presented.

Data from various onions were used. These have different cores depending on how they were formed. The cores could be nanodiamonds or voids and some onions have no core at all. Equation (7) was used in the comparison of these clusters.

We show the experimental results in Fig.5, after renormalisation of the results presented in paper [2]. Three sets of results are shown: 1) An onion, generated at $700^{\circ} \mathrm{C}$ which has concentric shells down to the centre and is depicted by black squares; 2 ) Onion 2 has a hollow core of $\approx 2.5 \mathrm{~nm}$ diameter, generated at $400^{\circ} \mathrm{C}$ and depicted by hollow circles; 3) Onion 3 generated at $730^{\circ} \mathrm{C}$ has a diamond core of $4.5 \mathrm{~nm}$ diameter and is depicted by open triangles. The exterior onions radii for these cases vary over the range $4-10$ 
nm. The dispersion of the curves shown in Fig.5 together with the changing spacings between the onion shells is in direct contrast to graphite which has a fixed interlayer spacing.

A striking coincidence between the numerical and experiment data for onion 1 is seen from Fig.5.

It is interesting to compare other results from X-ray diffraction experiments for the presence of variable-step onion fingerprints in nanodiamonds subjected to thermal annealing. It is easy to calculate mean values for the experimental data presented in Fig.5: for the open circles $\delta r_{\text {mean }}=$ $2.52 \pm 0.179 \AA$, for the grey circles $\delta r_{\text {mean }}=2.75 \pm 0.35 \AA$, for the black squares $\delta r_{\text {mean }}=2.68 \pm 0.26 \AA$, and for open triangles $\delta r_{\text {mean }}=3.082 \pm 0.11 \AA$. The corresponding doubled Bragg's angle calculated for the $C u K \alpha$ wavelength with equation (2) using $n=1$ are: $35.6^{\circ}, 32.54^{\circ}, 33.42^{\circ}$ and $28.97^{\circ}$, respectively. These values differ from the experimental values presented in Fig.1. However, the existence of a weak diffraction peak centred at $\sim 35^{\circ}$ given in paper [10] may be evidence for the existence of transitional forms of hybrid diamond-onion clusters arising from a crude nanodiamond substance subject to thermal annealing. These forms were investigated in more detail by electron microscopy in paper [2] and theoretically in papers [3], [1] as well as here.

The formation of COs during annealing may be considered as an inverse process to diamond formation. There are important processes that occur during annealing: 1) unfolding of adjacent (111) fragments of diamond planes during their transformation to fragments of (002) graphene planes; this process may appear in the course of enlargement of the lattice spacings from 
$\sim 2.2 \AA$ that is typical for diamond to $\sim 3.4 \AA$ that is typical for graphite; 2 ) welding the edges between the immediate fragments; 3)introduction of curvature to the planes through formation of short-range atomic ordering defects in the fragments. These defects may appear either during welding or as a result of the non-linear dynamics of atomic movement. Consequently defects of short-range-order atomic ordering may induce curvature of the fragments through topological alteration.

\section{Conclusions}

We conclude that the origin of the broadening of the XRD pattern of COs may reflect internal ordering of their shells similar to that which occurs for spirally twisted onions. The DFT study combined with analysis of experimental data confirms this conclusion. In addition a profusion of transitional allotropic forms of COs may exist between the ideal nanodiamonds and ideal onions. Some of them may be chiral and twisting.

[1] Ganesh P., Kent P. R. C., Mochalin V. Formation, characterization, and dynamics of onion-like carbon structures for electrical energy storage from nanodiamonds using reactive force fields. J. Appl. Phys. 2011; 110:073506-1-073506-8.

[2] Banhart F., Ajayan P. M. Carbon onions as nanoscopic pressure cells for diamond formation. Nature 1996; 382:433-435.

[3] Yastrebov S., Smith R., Siklitskaya A. Evolution of diamond nanoclusters in the interstellar medium. Mon. Not. R. Astron. Soc. 2010; 409: $1577-1584$. 
[4] Kenny S. D., Horsfield A. Plato: A localised orbital based density functional theory code. Comput. Phys. Comm. 2009; 180:2616-2621.

[5] Mykhaylyk O. O., Solonin Yu. M., Batchelder D. N., Brydson R. Transformation of nanodiamond into carbon onions: A comparative study by high-resolution transmission electron microscopy, electron energy-loss spectroscopy, X-ray diffraction, small-angle X-ray scattering, and ultraviolet Raman spectroscopy. J. Appl. Phys. 2005;97:074302-1-074302-1.

[6] McKenzie D. R., Muller D., Pailthorpe B. A. Compressive-stressinduced formation of thin-film tetrahedral amorphous carbon Phys. Rev. Lett. 1991; 67:773-776.

[7] Qin Z., MingZhi W., YanGuo Li, YuCheng Zh., LiangHua Z. Fabrication of onion-like carbon from nanodiamond by annealing. China Ser E-Tech Sci 2009; 52:3683-3689.

[8] Tomita S., Burian A., Dore J. C., LeBolloch D., Fujii M., Hayashi Sh.Diamond nanoparticles to carbon onions transformation: X-ray diffraction studies. Carbon 2002; 40: 1469-1474.

[9] Ida T., Shimazaki S., Hibino H., Toraya H. Diffraction peak profiles from spherical crystallites with lognormal size distribution. J. Appl. Cryst. 2003; 36:1107-1115.

[10] Osipov V. Yu., Enoki T., Takai K., Takahara K., Endo M., Hayashi T. et al. Magnetic and high resolution TEM studies of nanographite derived from nanodiamond. Carbon 2006; 44, 1225-1234. 
Figure captions.

Figure 1. The intensity profiles $P(2 \psi)$ measured for the most intense bands of the experimental X-ray diffraction patterns for $\mathrm{Cu}-\mathrm{K} \alpha$ radiation $(\lambda=1.54059 \AA)$. The double diffraction angle $\psi$ for the narrow peaked curve denotes the result for detonational nanodiamonds [5]. The broad peaked curve relates to nanodiamonds annealed in vacuo for $10 \mathrm{~min}$, at a temperature of $1500^{\circ} \mathrm{C}$, as reported in paper [5]. The solid line reproduces the Lorentzian fit (equation (4)) with the following set of parameters: $2 \psi_{B}=43.9^{\circ}, w_{L}=$ $2.338 \pm 0.0419^{\circ}, A_{L}=7.325 \pm 0.127$ arbitrary units. Black spots show the reference data for graphite.

Figure 2. The Lorentzian decomposition of the central part of the Xray diffraction pattern $P(2 \psi)$. Thin lines marked with numbers portray each of seven Lorentz contours used for the decomposition: the thick line shows the sum of all of the Lorentzians included (equation 5). The best fit parameters are: $w_{L 1}=w_{L 2}=w_{L 3}=w_{L 4}=w_{L 5}=w_{L 6}=w_{L 7}=1.994^{\circ}$; $2 \psi_{B_{1}}=19.900 \pm 0.023^{\circ}, 2 \psi_{B_{2}}=21.433 \pm 0.020^{\circ}, 2 \psi_{B_{3}}=22.753 \pm 0.020^{\circ}$, $2 \psi_{B_{4}}=23.987 \pm 0.021^{\circ}, 2 \psi_{B_{5}}=25.114 \pm 0.020^{\circ}, 2 \psi_{B_{6}}=26.188 \pm 0.021^{\circ}$, $2 \psi_{B_{7}}=27.368 \pm 0.024^{\circ} ; A_{L 1}=0.713 \pm 0.017, A_{L 2}=1.371 \pm 0.024, A_{L 3}=$ $1.834 \pm 0.031, A_{L 4}=2.307 \pm 0.040, A_{L 5}=2.814 \pm 0.045, A_{L 6}=2.348 \pm 0.044$, $A_{L 7}=0.977 \pm 0.034$, normalised units.

The parameters of the stochastic model, given in equation (6) for $i=1$ (dashed line)and $i=2$ (dash -dot line) are: $\psi_{o 1}=22.35 \pm$ $0.41^{\circ}, w_{1}=5.162 \pm 0.30^{\circ}, A_{1}=4.914 \pm 0.827, \psi_{o 2}=25.45^{ \pm} 0.079^{\circ}, w_{2}=$ $3.90 \pm 0.08^{\circ}, A_{2}=6.309 \pm 0.822$. 
Figure 3. A typical example of a stick and ball model of DFToptimised spiral CO. Green colour (colours are shown in the online version only) refers to the external atoms of the $\mathrm{CO}$; blue colour outlines the internal core of $C O$. The entrance to the CO is outlined by the red contour.

Figure 4. The cross section of the twisted CO presented in Fig.3. The full line marked with red (colours are shown in the online version only) shows an artist's reconstruction of the spiral ordering of atoms.

Figure 5. The dependence of the normalised radial distance of the COs on the intershell spacings. Grey filled circles (symbols) represent the calculated dependence using the equatorial cross sections of images of twisted COs simulated by MD/DFT at a temperature of $1135 \mathrm{~K}$ and presented in paper [3]. The black squares are the literature data for COs generated at $700^{\circ} \mathrm{C}$ and presented in [2]. Open circles represent the literature data for COs generated at $400^{\circ} \mathrm{C}$ and presented in [2]. The open triangles show the trend for an onion obtained at $730^{\circ} \mathrm{C}$. The solid lines connecting the symbols are for eye guidance only. 


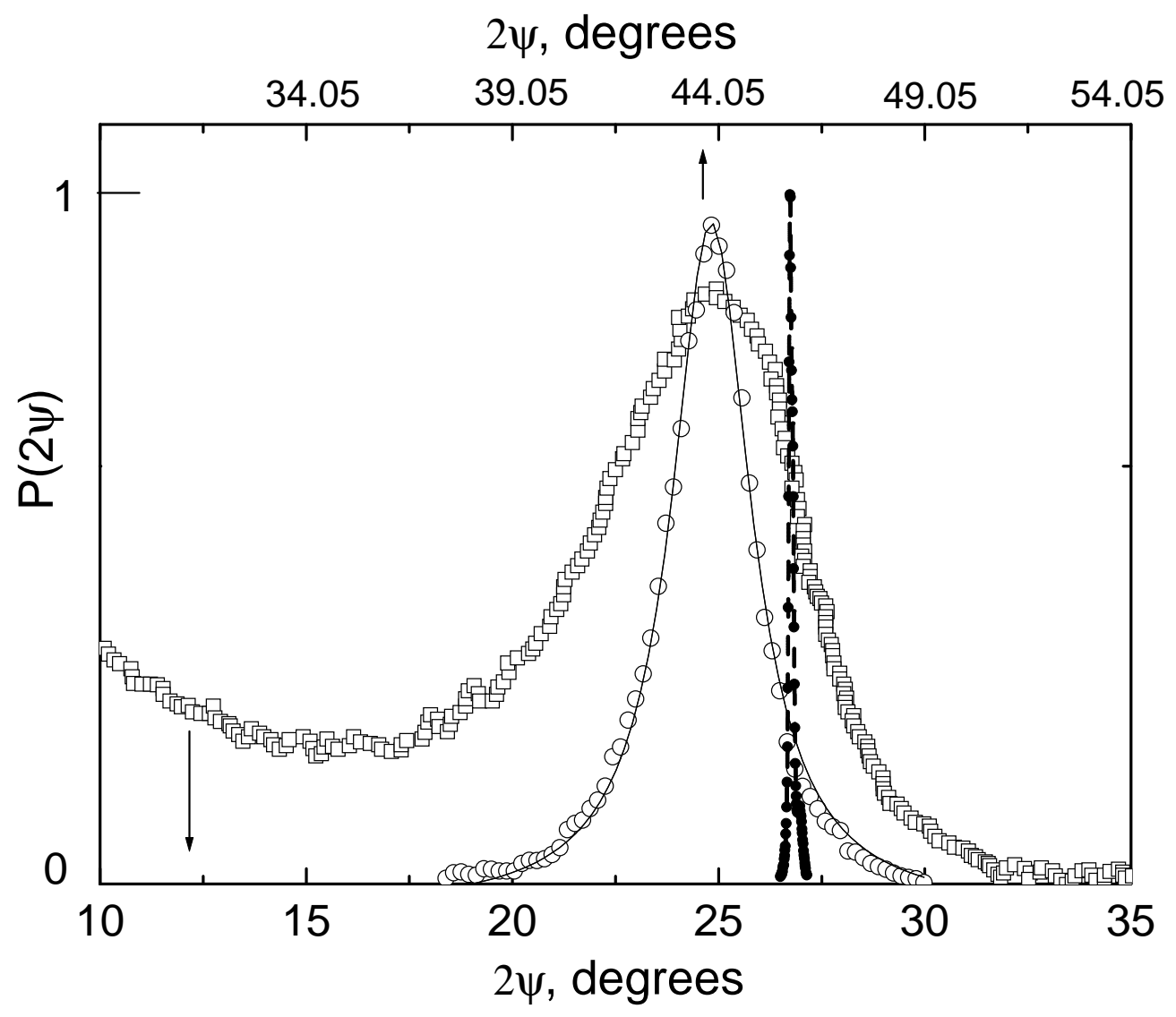

Figure 1: 


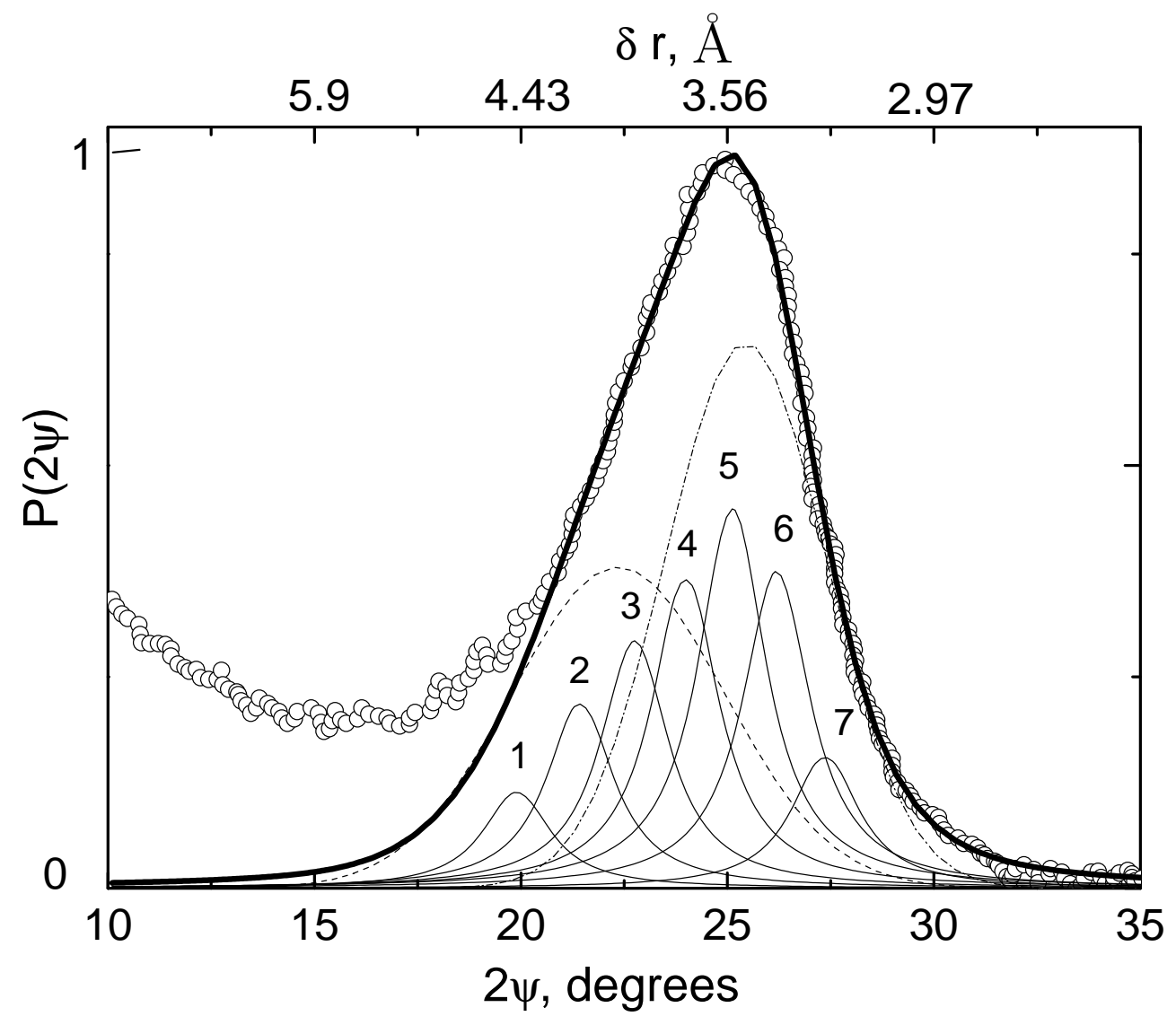

Figure 2: 


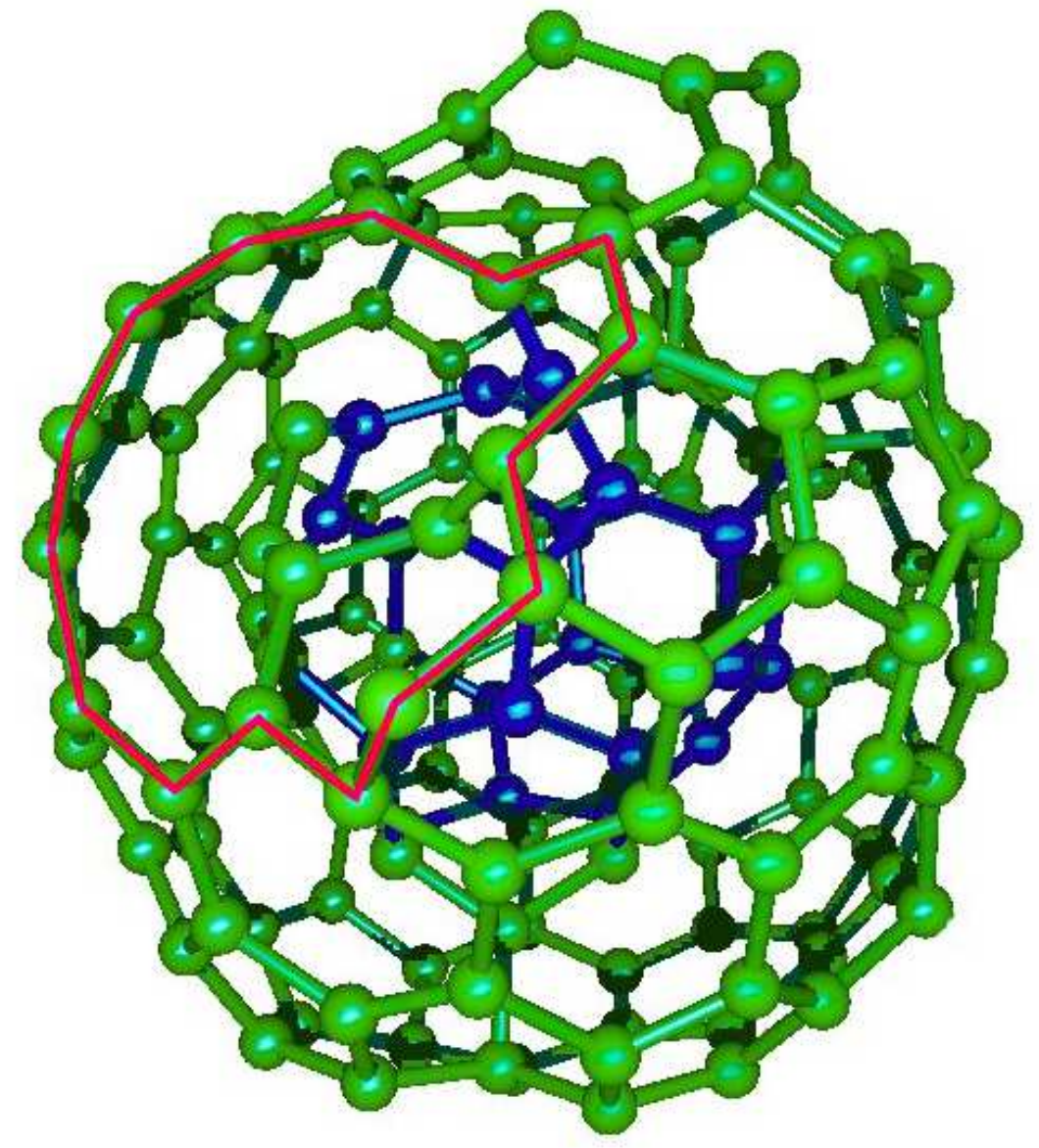

Figure 3: 


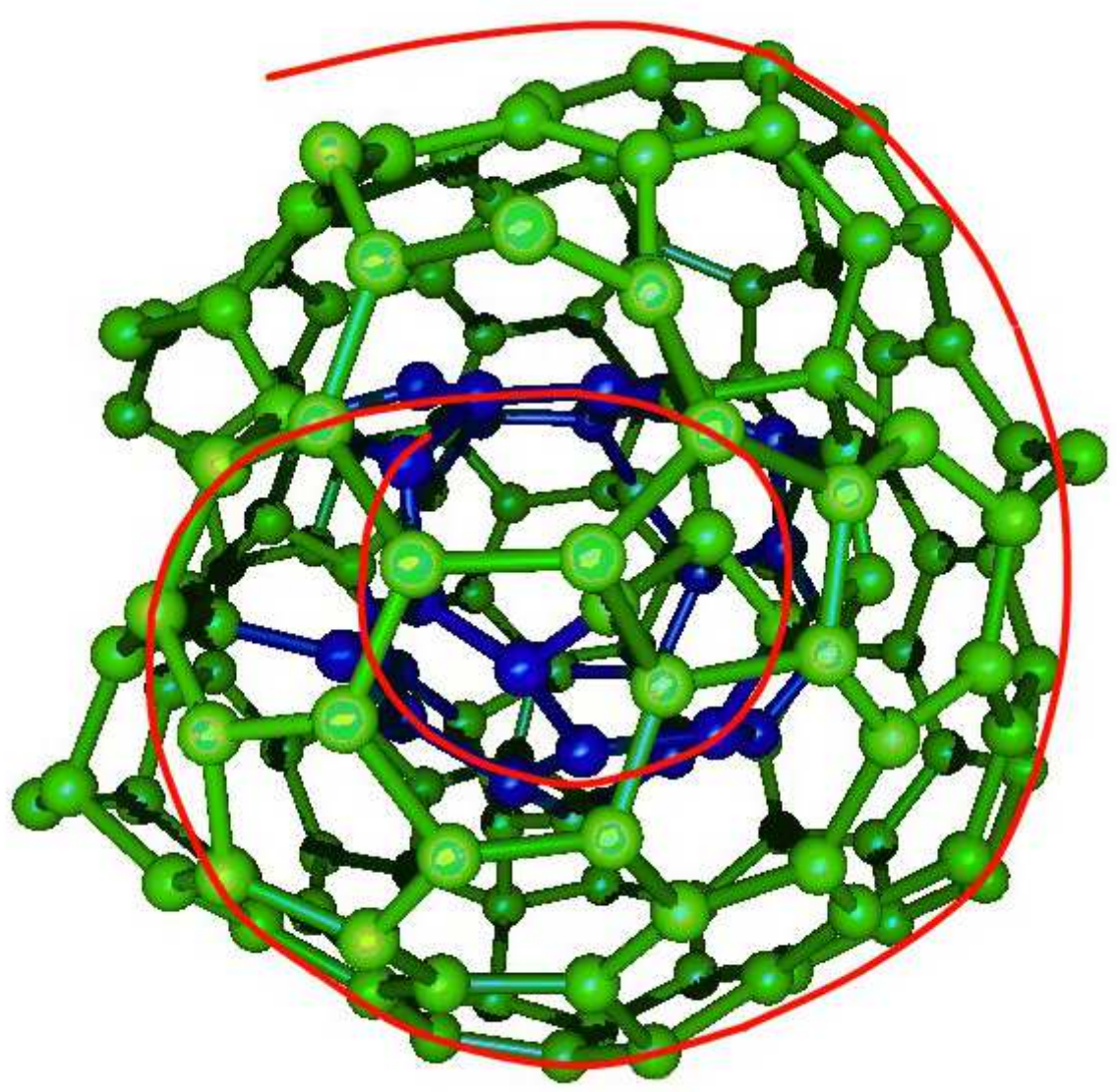

Figure 4: 


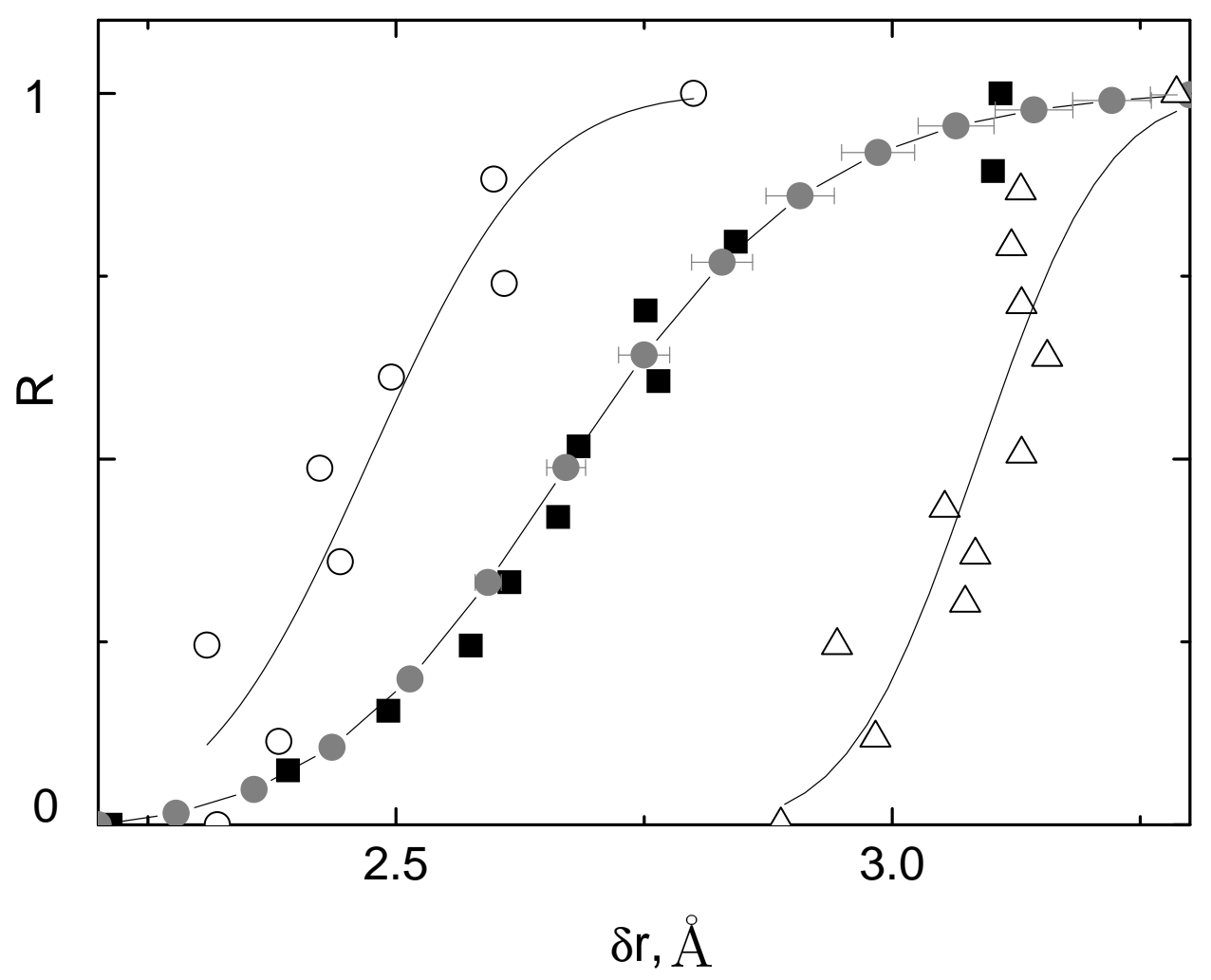

Figure 5: 